\section{Band 27, Heft 4, August 2011}

Editorial

264 Hereditäre Tumorerkrankungen des Gastrointestinaltrakts

Möslein, G.; Tannapfel, A. (Bochum)

Hauptthema

266 Lynch-Syndrom: Phenotypische und genotypische Heterogenität

Lynch, H.T.; Lynch, J.F.; Gatalica, Z. (Omaha); Boland, C.R. (Dallas)

275 Lynch-Syndrom - deutsche Erfahrungen Steinke, V.; Propping, P. (Bonn)

281 Familiäre adenomatöse Polyposis und andere Polyposis-Syndrome - humangenetischer Blickwinkel

Aretz, S. (Bonn)

290 Lynch-Syndrom, familiäre adenomatöse Polyposis und andere Polyposis-Syndrome - chirurgischer Blickwinkel

Schneider, C.; Gelos, M.; Möslein, G. (Bochum)

300 FAP-assoziierte und sporadische Desmoide Burdzik, E.; Quast, D.; Möslein, G. (Bochum)

309 Serratierte Läsionen im Kolorektum - ein pathologisches Problem oder ein Problem der Pathologen?

Baretton, G.B.; Aust, D.E. (Dresden)

316 Indexläsionen: Awareness-Alarm für Kliniker gibt es Precursor-Läsionen?

Schulmann, K.; Schmiegel, W. (Bochum)

322 Chemoprävention

Burn, J. (Newcastle upon Tyne)

Interdisziplinäres Gespräch

329 Hereditäre Tumorerkrankungen des Gastrointestinaltrakts

Möslein, G.; Riemann, J. (Ludwigshafen)

336 News / Ticker

337 Tagungen und Kongresse

340 Hinweise für Autoren

280 Impressum
Vol. 27, Issue 4, August 2011

Editorial

264 Hereditary Tumours of the Gastrointestinal Tract

Möslein, G.; Tannapfel, A. (Bochum)

Main Topic

266 Lynch Syndrome: Its Phenotypic and Genotypic Heterogeneity

Lynch, H.T.; Lynch, J.F.; Gatalica, Z. (Omaha); Boland, C.R. (Dallas)

275 Lynch Syndrome - Experiences from Germany Steinke, V.; Propping, P. (Bonn)

281 Familial Adenomatous Polyposis and Other Polyposis Syndromes - the Human Genetics Point of View

Aretz, S. (Bonn)

290 Lynch Syndrome, Familial Adenomatous Polyposis and Other Polyposis Syndromes - the Surgical Point of View

Schneider, C.; Gelos, M.; Möslein, G. (Bochum)

300 FAP-Associated and Sporadic Desmoid Tumours Burdzik, E.; Quast, D.; Möslein, G. (Bochum)

309 Serrated Lesions of the Colorectum - a Pathological or a Pathologist's Problem?

Baretton, G.B.; Aust, D.E. (Dresden)

316 Index Lesions: Awareness Alarm for Clinicians Do Precursor Lesions Exist?

Schulmann, K.; Schmiegel, W. (Bochum)

322 Chemoprevention

Burn, J. (Newcastle upon Tyne)

Interdisciplinary Discussion

329 Hereditary Tumours of the Gastrointestinal Tract

Möslein, G.; Riemann, J. (Ludwigshafen)

336 News / Ticker

337 Meetings and Conferences

340 Guidelines for Authors

280 Imprint

\section{KARGER}

Fax +497614520714

Information@Karger.de

www.karger.com (c) 2011 S. Karger GmbH, Freiburg 